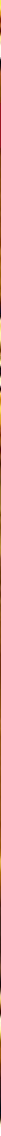

\title{
QUANTUM THERMODYNAMICS
}

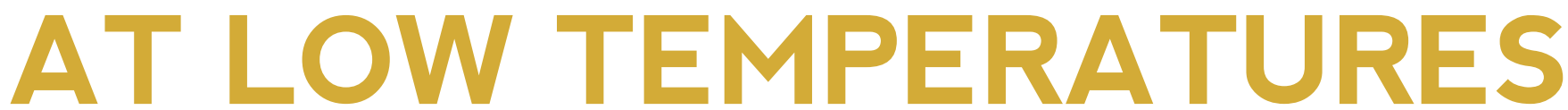

I Jukka P. Pekola - DOI: https://doi.org/10.1051/epn/2021302

$\triangle$ Pico group - QTF Centre of Excellence - Department of Applied Physics - Aalto University School of Science P.O. Box $13500-00076$ Aalto, Finland

Low temperature phenomena and methods are quantum thermodynamics per se. Modern engineered quantum systems, for instance those used for superconducting quantum information processing and mesoscopic electron transport, provide working media for realizing devices such as quantum heat engines and refrigerators and a testbed for fundamental principles and phenomena in thermodynamics of quantum systems and processes.

Quantum Thermodynamics?

Quantum thermodynamics presents an interplay and coexistence of quantum mechanics and thermodynamics, the two perhaps antagonist avenues in physics. It has existed as a field of science already for some time, most notably under the title "open quantum systems". This discipline describes genuinely quantum mechanical systems interacting with external world. The latter can often be considered as classical in nature, providing a heat bath or a collection of such baths. The position that we take here for describing quantum thermodynamics is to make it a synonym with "thermodynamics of quantum systems and processes".
Low temperature domain

Our focus in this article is the low temperature environment, typically in the sub-kelvin regime. Foundational developments, experiments and technology in low temperature physics, like the various refrigeration techniques, magnetic phenomena, thermometry etc. are to be considered quantum thermodynamics (much $\bullet \bullet \bullet$

$\triangle$ FIG 1: A photo of a typical experimental setup housing a superconducting quantum circuit. Sample holder with RF-connectors carries the chip in the middle. At the very centre, a cross-shaped transmontype superconducting qubit couples capacitively to coplanar wave resonators acting as harmonic oscillators. For a quantum thermodynamics experiment, e.g. in [4], the resonators are terminated by on-chip resistors (too small to see in this photo) that act as heat baths or absorbers of heat. The overall size of the square-shaped chip in the centre is $7 \mathrm{~mm} \times 7 \mathrm{~mm}$. (Photograph courtesy of Bayan Karimi.) 


\section{How to build quantum heat engines and refrigerators where the working substance obeys (nearly) coherent quantum dynamics? In this context, can one find "quantum supremacy" in form of higher powers and efficiencies as compared to classical reference devices?}

\begin{abstract}
- $\cdots$ before the name was coined), especially in the sub-kelvin temperature range [1]. One can thus say that quantum thermodynamics experiments have a century old history by now, ever since the cryogenic liquefaction of gases and discoveries of superconductivity.

Low temperatures provide a unique setting for quantum thermodynamics. Different physical sub-systems (phonons, electrons, nuclei among others) are typically weakly coupled to each other and their properties can be controlled and monitored individually, with relatively slow relaxation times amenable for experiments. But what brings yet another important twist to this story is the development of experimental techniques in the fields of microand nanofabrication, quantum information devices (e.g. superconducting qubits), and mesoscopic transport in electronic circuits. Local probing of particles, the quantum states and temperature are key ingredients for successful experiments. Activity and investments in these related areas have facilitated the emergence of a new field of research, the circuit quantum thermodynamics (cQTD).
\end{abstract}

v FIG 2: Mesoscopic single-electron boxes and transistors are next to ideal tools for stochastic thermodynamics experiments at low temperatures [2]. They allow for accurate determination of heat and work, and the experiments can easily be repeated many times, up to millions of repetitions, for collecting reliable statistics. Here a single electron box is monitored by a single-electron electrometer. By electrometer observation and feedback process the concept of Szilard's engine (lower inset), a form of Maxwell's demon, can be realized [3]. The main frame demonstrates apparent violation of the second law in the traditional sense: average work W done in a Szilard's engine cycle is negative because of the information gained by the observer (electrometer). (Figure adapted from [3].)

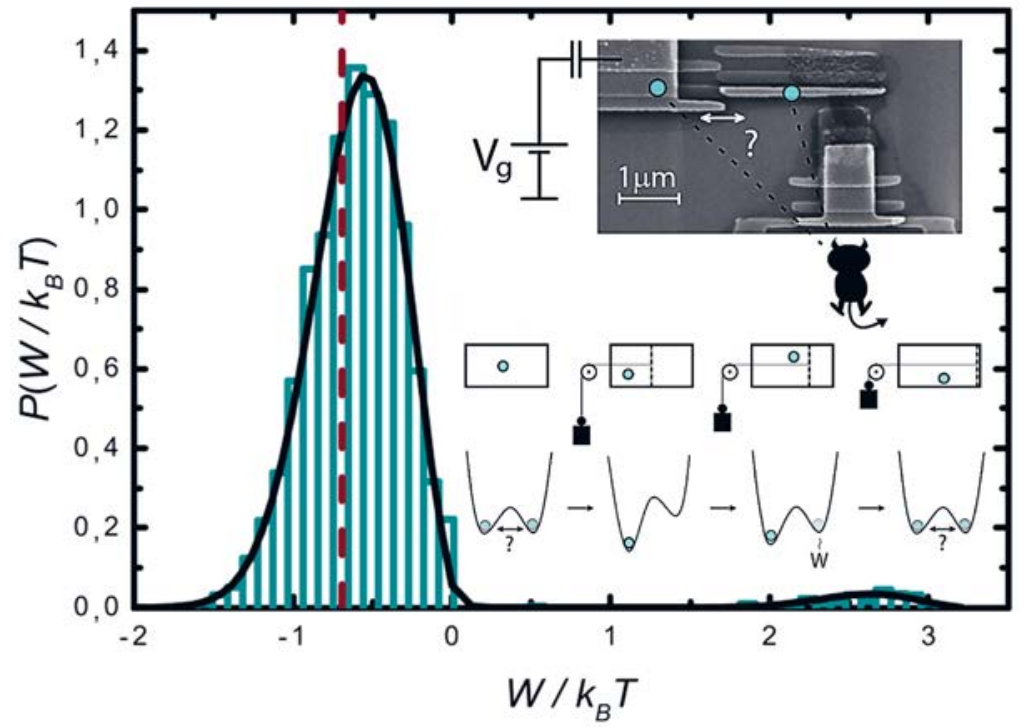

Selected toolbox for quantum thermodynamics experiments

Being a little biased one might say that the birth of quantum thermodynamics experiments at low temperatures took place with the stochastic thermodynamics experiments on quantum dot and single-electron box circuits [2]. Yet the "quantumness" of these experiments can be debated since their dynamics at low operation frequencies, as applied in the experiments, follows classical rate equations. The remarkable advantage of these setups is that the (charged) particles can be followed one by one with high resolution using ultrasensitive electrometers, and their energetics is governed by a simple Hamiltonian determined by the electrostatic charging energies. The outcome of these experiments was, first of all, verification of common fluctuation relations (relations that imply the first and second law of thermodynamics for stochastic processes) with unprecedented accuracy, and demonstrations of both non-autonomous and autonomous Maxwell's demons, shedding light to the almost two centuries old puzzle of the relation between information and energy [3]. The question of the minimal cost in computing was also addressed. This is the Landauer bound, where the energy cost equals Boltzmann constant $\mathrm{k}_{\mathrm{B}}$ times temperature $\mathrm{T}$ times $\ln (2)$, $\mathrm{k}_{\mathrm{B}} \mathrm{T} \ln (2)$, per bit in a quasistatic process.

The stochastic thermodynamics experiments by monitoring and counting particles (charges) are operating mostly based on classical physics. Besides this, they give only indirect information about heat and work, hinging on the model, in other words the Hamiltonian, of the system under study. True thermodynamics, on the contrary, counts on direct observations of heat currents and temperatures, and power consumption of the sources. To achieve these capabilities, our laboratory has been working over the years to realize on-chip electronic thermal detectors realizing bolometry and calorimetry with ultrasensitive thermometry. Bolometry here means detection of steadystate heat currents, whose sources can be Joule dissipation, injection of hot carriers, or coupling to photonic or phononic degrees of freedom in the circuit. Calorimetry, on the other hand, refers to thermal detection of single events, like absorption or emission of a photon in the element whose temperature is monitored. In both these tasks one needs a capable thermometer with proper figures of merit. The remarkable advantage of low temperatures in thermodynamic experiments originates from the ability to realize energy absorbers, say with only $10^{8}$ thermally distributed electrons in a metal, having an extremely small heat capacity (about $100 \mathrm{k}_{\mathrm{B}}$ or less) and extremely weak thermal coupling to the phonon heat bath. Measuring steady-state heat currents with thermometers is based on a very simple principle: in a linear system the temperature change is directly proportional to heat current via thermal conductance like in Ohm's law where voltage is proportional to the current via conductance. On the other hand, calorimetric 
principle is demonstrated in Fig. 3; currently its energy resolution in state-of-the-art experiments is already limited by the fundamental thermal fluctuations and not by the measurement apparatus.

We use thermal detectors for measuring energy dissipation in quantum circuits. Superconducting qubits based on standard Josephson junctions and resonators (harmonic oscillators) provide a basis for realization of a multitude of devices and experiments. Superconducting quantum technology has advanced enormously from the realization of the first superconducting qubits more than 20 years ago: this development offers us building blocks with versatile control options and very low internal dissipation and decoherence rates. By combining these circuits with the mesoscopic baths, i.e. the absorbers described above, one can then construct quantum heat valves, rectifiers, heat engines and refrigerators, and thermal masers, just to mention a few examples, and to address the scientific challenges described below.

\section{Scientific questions}

What are then the big questions in modern quantum thermodynamics? On a very general level, the question of how the laws of thermodynamics in the quantum setting are realized is an interesting issue. Classical stochastic thermodynamics stretches the traditional thermodynamics to a regime where fluctuations play an important role. Are the fluctuation relations governing these processes applicable to quantum systems, where the measurement and the measurement apparatus cannot be viewed as an innocent witness of what is happening in the system itself? How to measure work and heat in an open quantum system? One may also ask how to build quantum heat engines and refrigerators where the working substance obeys (nearly) coherent quantum dynamics? In this context, can one find "quantum supremacy" in form of higher powers and efficiencies as compared to classical reference devices? Currently there is no unanimous answer to this question: creating quantum coherences costs in general energy, i.e. one needs to do work to produce it, meaning that with the most obvious operation protocols coherent quantum dynamics is not useful. One topical objective in CQTD is how to build the most sensitive detector of energy exchanged between the quantum system and its environment? Ultrasensitive calorimetry is currently under intensive study, and it can eventually become the microscope of quantum dynamics on the level of exchange of energy by individual quanta emitted or absorbed by the quantum system. This would give us the optimal tool to investigate, e.g. the said stochastic thermodynamics in true quantum regime. Many other fundamentally and practically important questions arise and can potentially be answered by CQTD: for instance, where is the "Heisenberg cut", i.e. the boundary between the quantum system and the classical environment in a

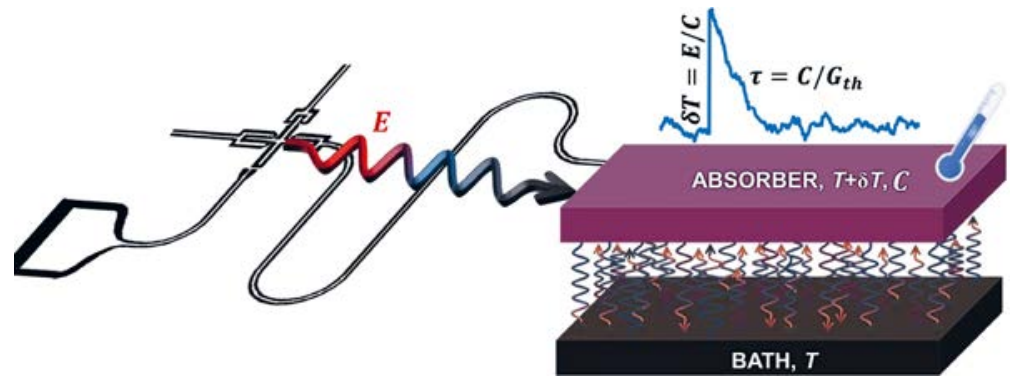

$\triangle$ FIG 3: One of the central tools in quantum thermodynamics is a thermometer measuring the heat absorbed in a mesoscopic bath. Here we depict the idea of a calorimetric measurement due to a single absorption event. A qubit (X-shaped object on the left) relaxes to its ground state and emits a quantum of energy via the resonator in the middle to the absorber on the right, a tiny metal electrode (not in scale). The thermometer observes this energy in form of instantaneous temperature rise due to finite heat capacity C and subsequent decay. The noise in the trace arises from fundamental thermal fluctuations and depends on $\mathrm{C}$ and thermal conductance $\mathrm{G}_{\mathrm{th}}$ of the absorber element [5]. (Figure by Bayan Karimi.)

realistic experiment? What happens when the couplings between sub-systems (inter-quantum system couplings, couplings to the heat baths) are tuned at will. Standard ways of treating open quantum systems are based on weak-coupling assumptions, where sub-systems can be identified and work and heat are definable: but once the coupling becomes sufficiently strong, such separation becomes meaningless, and one needs to revise the whole approach to the quantum thermodynamics problem. How does a quantum system thermalize, and does it find an equilibrium thermal state even in the absence of a heat bath?

With the tools at hand we are about to address many exciting fundamental physics questions at the crossroads of quantum mechanics and thermodynamics, and to build devices with unprecedented qualities and performance.

\section{About the Author}

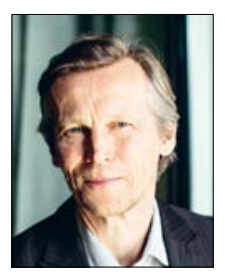

Jukka Pekola is a professor of quantum nanophysics at Aalto University in Helsinki, and directs the national centre of excellence, Quantum Technology Finland. He investigates thermodynamics and control of heat in quantum circuits.

\section{References}

[1] O. V. Lounasmaa, Experimental Principles and Methods below $1 \mathrm{~K}$ (Academic Press, London, 1974)

[2] J. P. Pekola and I. M. Khaymovich, Annual Review of Condensed Matter Physics 10, 193 (2019)

[3] Jonne V. Koski, Ville F. Maisi, Jukka P. Pekola, and Dmitri V. Averin, PNAS 111, 13786 (2014)

[4] Alberto Ronzani, Bayan Karimi, Jorden Senior, Yu-Cheng Chang, Joonas T. Peltonen, ChiiDong Chen, and Jukka Pekola, Nature Physics 14, 991 (2018)

[5] Bayan Karimi, Fredrik Brange, Peter Samuelsson, and Jukka P. Pekola, Nature Communications 11, 367 (2020) 